\title{
An auxiliary study of the smart grid deployment in India. Philosophy and key drivers
}

\author{
Farhan Beg \\ Department of Electrical Engineering, \\ National Institute of Technology, \\ Srinagar, India \\ and \\ Global Energy Network Institute, \\ 1088, 3rd Avenue, San Diego, California, 92101, USA \\ Email: farhan.beg@geni.org
}

\begin{abstract}
With an ever increasing population coupled with a perpetual depletion of fossil fuel resources and many generation sites attaining their ultimate capacity, India is in dire need of an up gradation of its electrical infrastructure. A much smarter approach towards the electrical grid is needed. This smarter approach technically referred to as the 'smart grid', promises an affordable electrical environment. This paper presents the journey of the Indian power sector through time, analyses various demand and supply scenarios which are mutually exclusive and also assesses various issues facing the Indian power system. This paper also highlights the need for ICT infrastructure concepts as well as various technologies involved to deploy a smart grid infrastructure and thereby leading to a conception of safety by means of design and modification resulting in an enhanced electricity management environment and hence leading to energy independence and an electrical grid that is much more reliable, secure, efficient, and greener.
\end{abstract}

Keywords: smart grid; renewable energy; micro sources; electricity management.

Reference to this paper should be made as follows: Beg, F. (2016) 'An auxiliary study of the smart grid deployment in India. Philosophy and key drivers', Int. J. Smart Grid and Green Communications, Vol. 1, No. 1, pp.38-49.

Biographical notes: Farhan Beg obtained his Bachelor's of Technology in Electrical Engineering from the National Institute of Technology, Srinagar, India and is a Power System Simulation Researcher at the Global Energy Network Institute, San Diego, USA.

\section{Introduction}

India had an installed power generation capacity of $210 \mathrm{GW}$ as on November 302012 , which is about 154 times the installed capacity in 1947 (1,362 MW). Electricity generation growth has been steadily increasing year after year, and in the year 2011-12 the generation was about 876.8 billion units of energy with a growth of $8 \%$ over the previous year. During the 11th plan the power sector made a considerable progress with 
a capacity addition of $58 \mathrm{GW}$ which was significantly more than the capacity commissioned in the previous plans.

However, the demand has been consistently outstripping the supply even as availability of power has increased due to substantial/ significant investments on the supply side. India faces the challenge of poor reliability and a not up to mark quality of electricity, leading to frequent load shedding situations in the country. India has witnessed substantial energy and peak shortages of $8.5 \%$ and $11 \%$ respectively in the year 2011-12 (Moslehi and Kumar, 2010).

Figure 1 Indian electricity generation by type, 2000-2012 (see online version for colours)

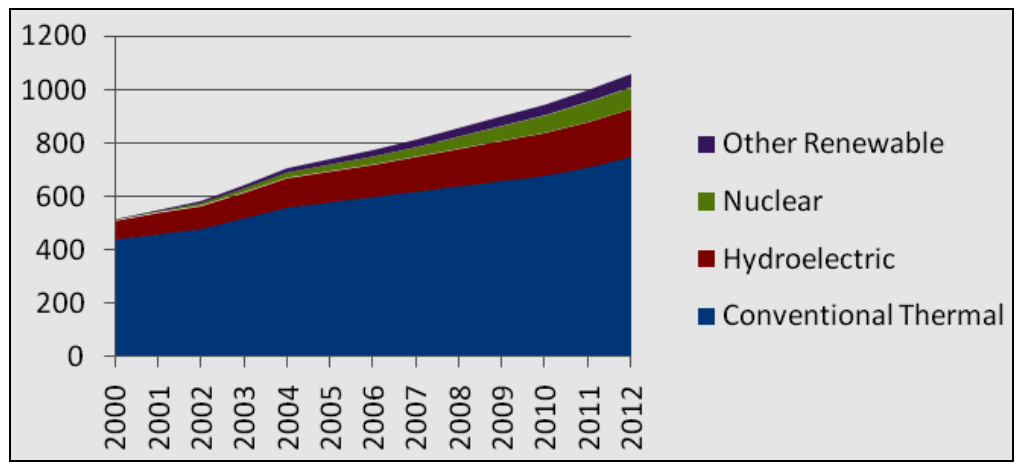

Source: Beg (2013); data from the IEA India energy report 2013

On July 31 2012, India saw itself under the grip of the largest electrical blackout in history bringing under its effect nearly 670 million people or roughly $10 \%$ of the world's population. The Northern Power Grid that connects three of the country's grids discontinued operating and ceased to function as blackouts extended almost 2,000 miles, from India's eastern border with Myanmar to its western border with Pakistan. Such an enormous power failure would pave way for more stringent regulations that need to be adhered to in truest sense to the chain of electricity delivery system in the power sector. Indian authorities are leaving no stone unturned to augment the supply chain of electricity delivery system so as the fragile and weary electrical power delivery system of India is mended to the extent that such blackouts do not occur in the near future. The primary issue is of adaptability if the way blackouts occur in India is compared with that of some other country where electrical supply is far more reliable. The Indian electrical system is conventionally rated as a fragile and an unreliable system lacking considerable self healing and progressive capabilities that tend to have a cascading effect on the overall health of the electrical system (Hodge and Milligan, 2011). Issues facing India's electrical system have been discussed as they are the key drivers for smart grid deployment in the country.

\section{Prognosis and smart grid introduction}

In an axiomatic sense, India's electric power framework that has served the populace for a long time - known as 'the grid' is finally running up against its limitations. Grids capabilities in the present developing socio economic scenario are not to be taken for 
granted. The establishment of the present electric power system dates back to over a 100 years when the sole purpose of the power system was to evacuate power from generating stations employing the use of coal to residential consumers that needed the electricity mostly for lighting purposes. Power generation was localised and was built around communities. With the advancement of technology and industry the needs of the people grew and leading to the generation sites to grow to supply the needed power. But with the increasing size of the electrical grid, optimisation and complete control cannot be satisfied by merely increasing the number of generation sites. Today the electrical power system delivers power to agriculture industry, commercial and residential consumers, trying miserably to cope up with the ever growing demand (Kaur, 2010). Systematically the hazards accompanied with relying on an overburdened grid grow in size, scope and convolution with every moment elapsed. The present limited one way interaction makes it difficult for the grid to respond to the ever changing and rising energy demands of the 21st century (Beg, 2013). To do justice to the Ministry of Power's 'Vision 2027' involving transformation of the Indian power sector into a secure, adaptive, sustainable and digitally enabled ecosystem by 2027 that provides reliable and quality energy for all with active participation of stakeholders, the power infrastructure needs to be upgraded.

Figure 2 Smart grid provides a two way communication (see online version for colours)

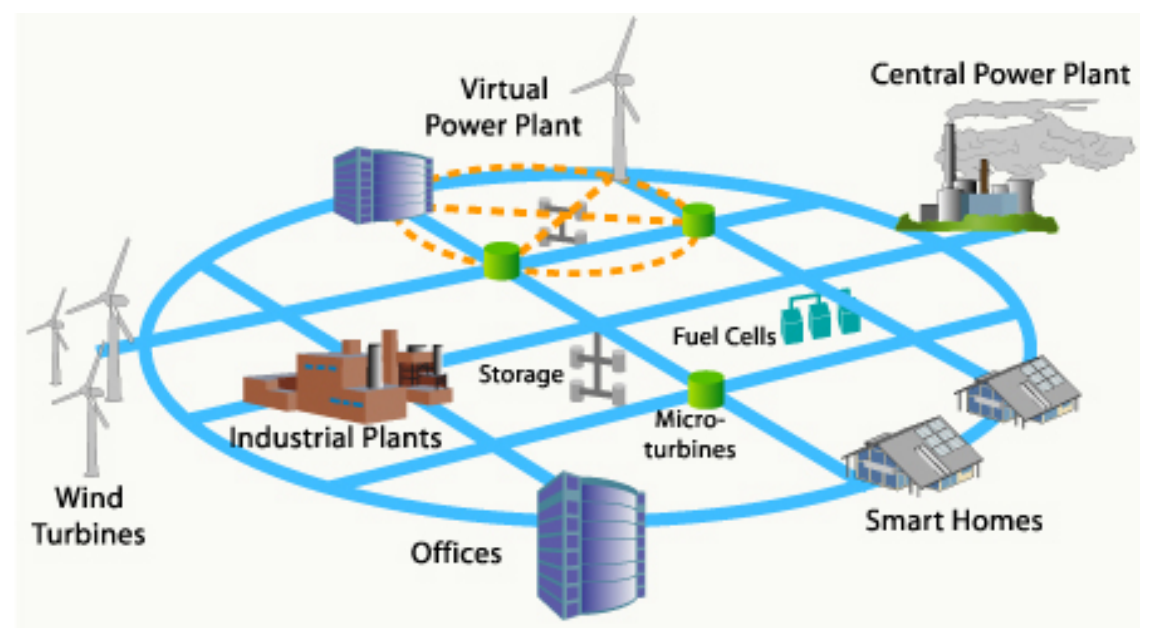

Source: http://www.powergenasia.com/conference/smartmeter.html

\section{Smart grid philosophy and associated technologies}

We are at the crossroads of a great transformation in the way electricity was perceived traditionally. A revolutionary makeover from a central, utility controlled network to a grid that is less central and more interactive with the consumers is on cards. The up gradation to a smarter grid promises to widely affect the industry's entire business model that consisted of vertically integrated entities and its relationship with all stakeholders in the chain affecting and involving utilities, energy service providers (ESP), regulators, technology and control dealers and all beneficiaries of the electric power. 
A smart grid makes this up gradation possible by bringing together the various concepts, philosophies and technologies in a common spectrum enabling an inter connection between the utility and the electric grid. Simply put, a smart grid is the integration of information and communication technology (ICT) into the electric transmission and distribution (T\&D) networks delivering electricity to consumers using a two way digital technology to enable a more efficient management of consumers end uses of electricity as well as a more efficient use of the grid to identify and correct supply demand imbalances instantaneously and detect faults in a 'self-healing' process that improves service quality, enhances reliability, and reduces costs (Beigh and Koul, 2013).

Several of the initiatives that have already been introduced into the system include supervisory control and data acquisition (SCADA), distribution management system (DMS), distribution automation system (DA), energy management system (EMS), automated meter reading (AMR), outage management system (OMS), enterprise resource planning (ERP) and geographical information system (GIS) (Novosel, 2008). Installing latest technology and systems in the power system promises a decrease in loss levels and a subsequent increase in the reliability of the network. Successful implementation of the smart grid would also require introduction on wide area measurement system (WAMS)based technology for achieving grid performance. Installation of phasor measurement units (PMU's) at the utilities is a prerequisite for WAMS. The existing interface involving SCADA/EMS-based grid operation has the potential to provide the steady state view of the power grid only. Dynamic real time measurements and visualisation of the power infrastructure which are useful for an optimal working of the grid as well as introducing corrective measure can be realised only with the introduction of PMU-based technology (Molderink et al., 2009).

\subsection{Network operations}

The main difference between the traditional power infra-structure and the smart grid is in the distribution area at the customer/network interfaces. However, in the areas of generation and transmission the role the system operators play will become quite complicated and critical as it will have to ensure an efficient, reliable system as well as the integration of other sources of energy. The smart grid will be critically system operators configured. An ancillary support system will be required from sophisticated and technologically advanced EMSs to oversee and manage all available energy resources and transmission parameters in every part of the system under a broad variety of operating conditions and possible future scenario (Tsoukalas and Gao, 2008).

\subsection{Automated metering infrastructure through smart meters}

Smart metering is a very important focus area for distribution utilities. The main demand driver for this upgradation is the energy accounting backbone created under the Restructured Accelerated Power Development and Reforms programme (RAPDRP). Bringing into action the vision of introducing a smart grid infra-structure, the industry can expect a rise in the demand for metering technologies such as AMR and prepaid metering. Smart meters will be installed at the customers premises to enable a bi-directional communication between the meters and the utility meter data management system (MDMS) (Huang et al., 2011). This two way communication will enable utility to understand the demand profile of the customers in near real time. The real time data also 
allows the customers to monitor and control their consumption. Implementation of tariff of day (TOD) functionality incentivises customers for demand side management. Smart metering also enables the remote connect and/or disconnect of erroneous customers thus introducing a transparent mechanism and ultimate control. A MDMS is required to be implemented to interface the system application and products-industry specific solutions-utilities (SAP-ISU, http://sap.ittoolbox.com/documents/sap-isu-a-briefoverview-14189) billing system (Sauter and Lobashov, 2011). This system obtains the meter data from the smart meters and is used for various purposes like billing, planning and load forecast etc. (Hart, 2008). The platform for deployment of the automated metering systems stresses on the need for the implementation of an advanced metering infrastructure (AMI) in India which presents a few challenges:

- Smart meters are a relatively new concept in the Indian electricity infrastructure and there are a few technical challenges with respect to their customisation for Indian conditions such as security protocols as well as there are no standards yet formulated for them.

- Suppliers are not yet prepared to bring smart meters in the Indian market as the technical regulations are not yet harmonised between the utilities and the suppliers and also because of a lack of skilled resources.

Figure 3 AMI systems (see online version for colours)

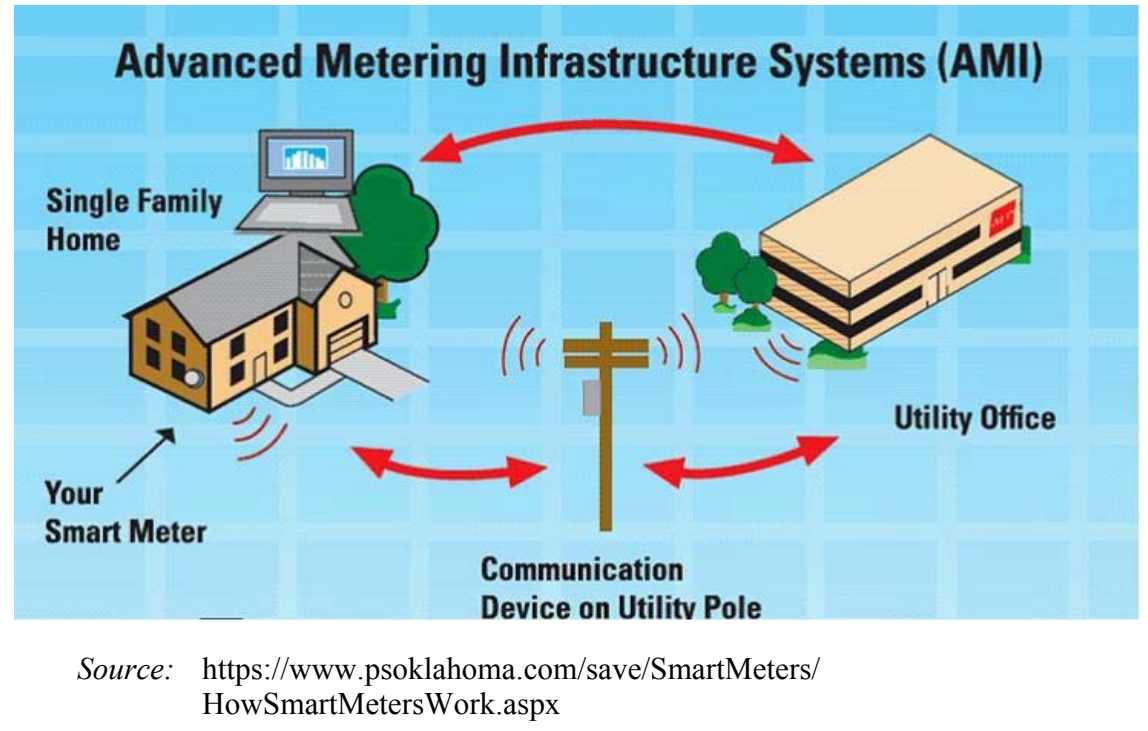

\subsection{Home area network}

Even though home area network (HAN) is not a primary driver of implementing the smart grid in India but it provides a definite edge to the idea of complete overhaul of the traditional power system to a grid of the future. A HAN provides the platform for smart devices in a home to communicate directly with the grid and enables the consumers to manage their electricity usage by measuring a home's electricity consumption more 
frequently through a smart meter; utilities can provide their customers with much better information to manage their electricity bills (Misra et al., 2013).

Figure 4 HAN visualisation (see online version for colours)

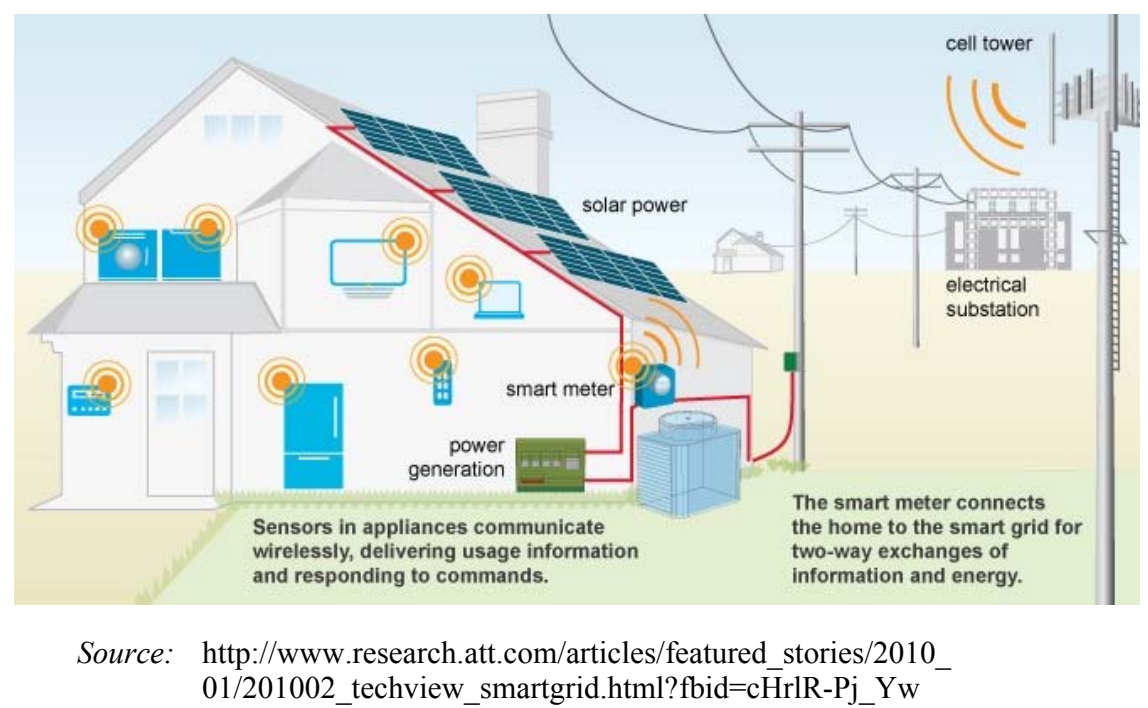

Inside the Smart Home, a HAN connects thermostats, refrigerators and other electrical devices to an EMS. Smart appliances and devices will adjust their run schedule to reduce electricity demand on the grid at critical times and lower consumer's energy bills (Tomich, 1998).

\subsection{FSAS for integration of renewable generation}

Integration of renewable energy in the grid is one of the biggest thrust areas for infrastructure up-gradation. The installed generation capacity of renewable generators is expected to grow manifold in the near future. India has set itself ambitious plans to achieve high generation targets in the next ten years. Considering the high variability and unpredictability of generation from renewable, the injection from renewable sources can be safely absorbed in the grid if the frequency in the grid is maintained in a comfortable range. Frequency support ancillary service (FSAS) can be used to complement the diurnal changes in the renewable generation. In future, based on the renewable forecast for the next day, a dispatch schedule for FSAS can be prepared such that the variation in the renewable generation can be absorbed easily. FSAS can thus also be used as mechanism to facilitate renewable integration by reducing the impact in the variation (Mohd et al., 2008).

Taking into account the restrained distress of global warming, the importance of distributed generation (DG) from cleaner sources such as wind and solar is critical. The Ministry of New and Renewable Energy Sources (MNRE) has proposed that to integrate renewable energy sources such as wind and solar energy at grid operator level will require a superior EMS and is to be converged at the grid operator level. The smart grids superior capability of introducing new sources of energy to the grid clearly signifies that 
more DG can be integrated into it. Solar and wind energy are crucial and primary factors of a cleaner and greener energy future.

\section{Key drivers of the smart grid in India}

India is relatively at a preliminary stage of its smart grid deployments with many technologies such as advanced metering, still at a very crucial juncture and its deployment being reviewed and is being tested at certain pilots identified in the country. Such technologies are undergoing various trials and advanced testing before they could be deployed all over the country. The coherence of many factors would drive India's adoption of smart grids such as reducing all technical and commercial losses and thus aiding to resolve the ceaseless supply demand gap. India is heading to upgrade its infrastructure to a more advanced electricity supply solution and hence flatter its sustainable, low carbon high growth economic development goals.

Certain factors will drive the adoption of the smart grid in India:

\subsection{Supply shortfalls and unrelenting increase in demand}

Demand especially the peak demand of electricity is continuously outpacing the India's Power supply. With an unmatched growth of the economy and a prosperous industrial arena with more and more households affording more and more electrical appliances leading to a phenomenon enhancement in the demand of electricity. Industries also require a consistently increasing power supplying to match the peaking curves of the industrial growth. Officially India is falling short by around $12 \%$ for energy demand while as $16 \%$ for peak energy demand. Addressing the needs of the growing economy and satisfying the needs of a developing society is the primary drivers of the adoption of smart grid technologies in India which could provide the required platform for renewable integration. In India's high growth economy the demand for electricity is forecasted to grow by about $10 \%$ per year as long as the already present gap is not closed.

\subsection{Distributed energy resources and subsequent loss reduction}

A Smart Grid can make a substantial contribution towards the reduction of losses by allowing integration of distributed energy resources (DERs) that can allow localised generation, thus reducing the transmission and equipment losses. A smart grid allows system operators to integrate local renewable energy sources which cannot only supply energy needs to the local community but also supply power to the grid thus offering a launch pad for the utilisation of natural local resources for the benefits of power supply leading to cost reduction. Both DG and distributed energy storage (DES) are encompassed in distributed resources (DR) (Chalkiadakis et al., 2011). A distributed utility uses both DR as well as load management to accomplish its goal. Integration of DG into an existing structure results in several benefits. These benefits consist of reduced line losses, reduction in environmental ramifications, peak saving, increase in the energy efficiency, T\&D congestion management, harmonic voltage support, and investment fluidity to upgrade existing generation, transmission, and distribution systems. Consumers also get benefitted from DG in terms of obtaining a better quality of supply at reduced costs (Brown et al., 2001). 


\subsection{Peak load management}

Managing peak load is another key driver for smart grid deployment in India. Energy cannot be stored for long duration in large scale and has to be increased to take care of its demand or demand reduced significantly by load shedding. A smart grid allows more 'perceptive' load control, directly or indirectly by controlling the appliances switching off the less important ones so as to reduce the usage to prevent peak time deficit and hence preventing the outages and load shedding. This can be done by either communicating to the consumer about the economic pricing incentives in a dynamic manner or by having a real time protocol to allow utilities have a direct control on the devices and setting less important tasks for a time when electricity is cheap and hence acting as a buffer for fluctuations in the peak energy supply (Budka et al., 2010). By incentivising the operation of appliances the consumer can control the amount of load they are using in a better way. Such measure can to an important extent mitigate the supply-demand gap. By introducing the right price signals and smart appliances/ devices, for example - a smarter grid can definitely reduce the need for infrastructure while keeping electricity affordable and reliable. During peak demand, the intense stress on the power system threatens its feasibility and reliability and raises the possibility of widespread blackouts.

\subsection{Micro grids deployment}

Micro grids focus on the localised generation and distribution of electricity using the grid infrastructure that either operates in the island mode or integrated with the grid. A smart microgrid would provide the intelligent last mile communication pathway for issues such as demand forecasting, detecting and eliminating electricity theft, localising and islanding faults as well as remote monitoring of the infrastructure.

A smart microgrid would help the locally installed renewable generation to be integrated with the conventional power supply. The captive generation can be customised for local energy demand curve. DG also eliminates the need to supply subsidised power to rural areas during peak load (Hayes et al., 2011).

A smart prepaid meter can help provide a highly dynamic electricity distributing mechanism, by which power is paid for before consumption. The grid is managed remotely wherein a smart central communication system monitors and collects data from all meters. On the consumption side load limit can also be configured remotely and a variable pricing scheme implemented for different types of loads viz. household, commercial and telecom.

\subsection{Renewable energy}

The Indian renewable growth has been performing a constant steep increase in the past couple of decades. Even though a significant portion of the contribution of RES to the power generated is from the hydro power with almost $20 \%$ still being obtained from this sector, it has not progressed much on utilising the other renewable sources of energy. Recently Indian power sector has started foraging into other renewable energy sources. Wind power being the most prominent face of renewable energy for the past decade or so. Since 2010 significant activities in a few other renewable energy sectors such as solar have also been observed. With the inception of the industrious National Solar Mission in 2010 and the consequent successful operation of the initial stages the Indian Government 
has shown its eagerness in harnessing the significant potential that solar energy presents for India. India can also utilise other renewable energy sources such as small hydro and biomass and there is a huge potential for it. Some segments that show little activity currently - like wave, tidal and geothermal, for instance - have the potential for significant growth in future. As many of the renewable energy sectors are nascent, there is significant need and eagerness from Indian corporate - for technology transfer from companies who are at the cutting edge of clean technology. To successfully integrate clean power from renewable sources such as wind and solar energy, utilities will need a smart grid to manage problems caused by intermittency (the sun and wind only happen during certain times of the day) and distributed power (Chakraborty et al., 2012). Stirred by the need to protect the environment and the desire to tap in all the possible sources of power, this move would also be a primary smart grid driver.

\subsubsection{Wind energy in India}

The wind power programme in India was initiated in 1983-84 aimed at speeding up the commercialisation of wind power programme in the country and has progressed rapidly in the last few years. The relatively short period required for the installation of wind turbines and the constant advancements made in the wind turbine technology leading to higher reliability and performance of the turbines has made wind power the most favourable choice of capacity additions in the country. The latest estimates indicate the total potential of wind energy in the country to be at $45,000 \mathrm{MW}$. The wind sector of the country has observed significant investments, catalysed by the highly indicative wind potential, availability of wind farm equipment at subsidised rates and favourable government policies. Presently, India $(15,700 \mathrm{MW})$ is at the fifth position with China (44,733 MW), the USA (40,180 MW), Germany (27,215 MW) and Spain (20,676 MW) ahead of it (Ackermann, 2005). Wind power accounts for about $8 \%$ of India's total installed power capacity.

Figure 5 Wind power instalment in the country (see online version for colours)

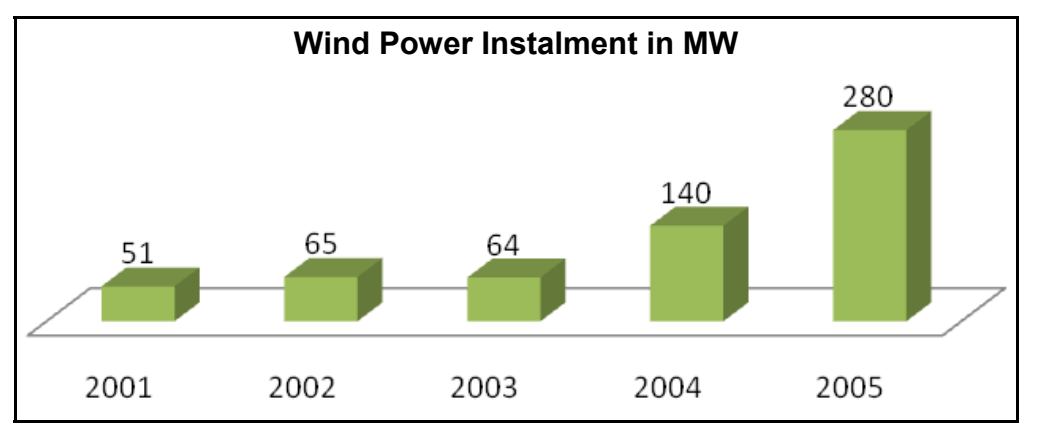

Source: Beg (2013); data from IEA wind country wise wind reports

Historically, wind energy has always met and many a times exceeded the targets like in the 10th plan (2002-2007) and the 11th plan (2007-2012) periods. During the 10th plan period the target set was of 1,500 MW whereas actual installations were about $5,427 \mathrm{MW}$. Much similarly in the 11th plan the revised target was for 9,000 MW and the actual installations tended to the higher side at 10,260 MW. In 2011, the Centre for Wind Energy Technology, a state run organisation assessed India's Wind power potential as 
$102,778 \mathrm{MW}$ at 80 metres height at $2 \%$ land availability significantly higher from the earlier estimates of approximate $49,130 \mathrm{MW}$ at 50 metres height also at $2 \%$ land availability. Different research organisations utilise different models for mapping the wind resource (Foley et al., 2012). The Lawrence Berkeley Centre in a study assuming a turbine density of $9 \mathrm{MW} / \mathrm{square} \mathrm{km}$, concluded the total wind potential in India with a minimum capacity factor of $20 \%$ ranges from $2006 \mathrm{GW}$ at 8 metre hub height to 3,121 GW at 120 metre hub height (Fan et al., 2009).

\subsubsection{Solar energy in India}

The Indian subcontinent is blessed with a rich potential of solar energy resource. The average intensity of solar radiation in India is of the order of $20 \mathrm{MW} / \mathrm{square} \mathrm{km}$. India has a geographical area of 3.287 million square $\mathrm{km}$, and hence the total solar energy potential tends to as high as 657.4 million MW though only $12.5 \%$ of the land area which is about 0.413 million square $\mathrm{km}$ can be installed with solar energy platforms because of logistical and theoretical reasons. Even if $10 \%$ of the area is used, the available solar energy can amount to nearly 8 million MW per year which is purely for the basis of analysis because the conversion efficiency of solar energy is low and the energy actually available will be lower than the estimated values.

Figure 6 Indian solar PV growth (see online version for colours)

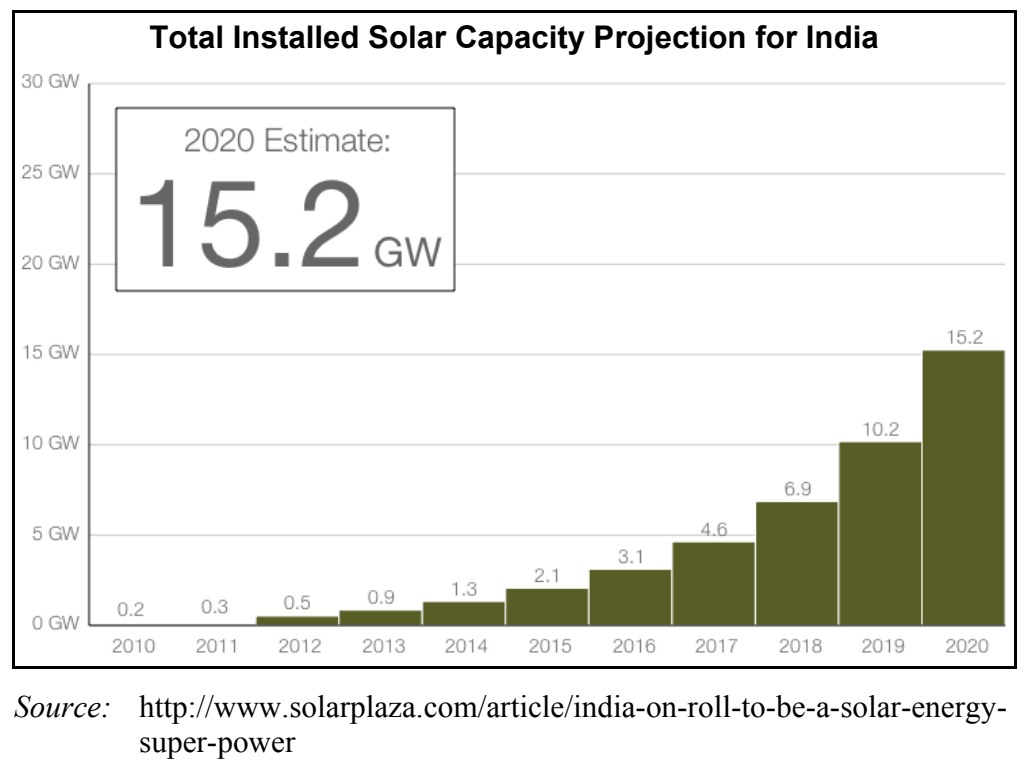

Up until now, solar energy has remained dormant in the Indian energy scenario. The total on-grid capacity has reached $941.48 \mathrm{MW}$ while as the total potential of solar power is 50,000 MW. Assertively, India's solar energy exposition is headed towards a rise, banking on the widening demand-supply gap, continual increase in the non-renewable fuel prices, the National Solar Mission, various local initiatives, renewable energy allocations including the solar energy allocation as well as advancements in cheap and highly practical technologies. India is enroute to come up as a solar energy hub banking on its solar potential. 


\section{$5 \quad$ Large scale integration of wind and solar energy in the grid}

Integrating wind and solar energy into the grid can allow the grid to be far more reliable, independent, efficient and self remedial in the case of power system breakdown and blackout; allowing clusters of localities to receive power from the local renewable generation and hence lead to a grid transition from a non-self-healing infrastructure to a detect and control infrastructure (Cecati et al., 2011). This integration to a large extent is directed towards managing peak loads, offering new services to meet user needs at an individual level and improving asset use. It involves a systems approach to develop and demonstrate the technical, regulatory, economic and other barriers in the use of renewable energy and DG The major scope of integration of renewable energy is of wind and solar in India and while as such a proposition is already underway, wind and solar energy both suffer from a technical issue of intermittency, a variability that cannot be controlled, unpredictability to some extent and location dependency (Liserre et al., 2010).

\section{Conclusions}

While as the paper focused on the review of the smart grid technologies that can be deployed for the inception of an efficient and reliable electrical grid infrastructure a study on the key drivers of the smart grid deployment for policy argument was also included. Smart grid can provide a solution for a complete overhaul of the electrical grid and the transition from the traditional grid to a smart grid can only occur when the platform is prepped for the deployment of smart grid technologies.

This paper analysed the need of introducing smart grid technologies and also presented a general introduction of their prospects. Also while as this general study can be used to motivate a more research related to application of information architecture concepts of a smart grid, it can also act as a pioneer to establish an information communication and technology-based network in general and a smart grid domain in particular. Also this paper can act as a foundation for further research that can be conducted on the feasibility of the application of ICT technologies in certain particular pilots whose case study has to be conducted separately.

\section{Acknowledgements}

The author would like to thank Mr. Peter Meisen, founder of Global Energy Network Institute, USA, for his valuable insights and suggestions.

\section{References}

Ackermann, T. (Ed.) (2005) Wind Power in Power Systems, Vol. 140, John Wiley, Chichester, UK. Beg, F. (2013) Integrating Wind and Solar Energy in India for a Smart Grid Platform.

Beigh, F. and Koul, A. (2013) 'An ancillary review of ICT based technologies in the road safety paradigm', International Journal of Information, Vol. 3, No. 5, pp.188-192.

Brown, R.E. et al. (2001) 'Siting distributed generation to defer T\&D expansion', Transmission and Distribution Conference and Exposition, 2001 IEEE/PES, Vol. 2. 
Budka, K.C. et al. (2010) 'Communication network architecture and design principles for smart grids', Bell Labs Technical Journal, Vol. 15, No. 2, pp.205-227.

Cecati, C., Citro, C. and Siano, P. (2011) 'Combined operations of renewable energy systems and responsive demand in a smart grid', IEEE Transactions on Sustainable Energy, Vol. 2, No. 4, pp.468-476.

Chakraborty, A. et al. (2012) 'Integrating statcom and battery energy storage system for power system transient stability: a review and application', Advances in Power Electronics, pp.1-12.

Chalkiadakis, G., Robu, V., Kota, R., Rogers, A. and Jennings, N.R. (2011) 'Cooperatives of distributed energy resources for efficient virtual power plants', The 10th International Conference on Autonomous Agents and Multiagent Systems, International Foundation for Autonomous Agents and Multiagent Systems, May, Vol. 2, pp.787-794.

Fan, S. et al. (2009) 'Forecasting the wind generation using a two-stage network based on meteorological information', IEEE Transactions on Energy Conversion, Vol. 24, No. 2, pp.474-482.

Foley, A.M. et al. (2012) 'Current methods and advances in forecasting of wind power generation', Renewable Energy, Vol. 37, No. 1, pp.1-8.

Hart, D.G. (2008) 'Using AMI to realize the smart grid', Power and Energy Society General Meeting - Conversion and Delivery of Electrical Energy in the 21st Century, IEEE.

Hayes, C.C. et al. (2011) 'Intelligent support for product design: looking backward, looking forward', Journal of Computing and Information Science in Engineering, Vol. 11, No. 2, pp.131-146, 021007.

Hodge, B. and Milligan, M. (2011) 'Wind power forecasting error distributions over multiple timescales', Power and Energy Society General Meeting, IEEE.

Huang, A.Q., Crow, M.L., Heydt, G.T., Zheng, J.P. and Dale, S.J. (2011) 'The future renewable electric energy delivery and management (FREEDM) system: the energy internet', Proceedings of the IEEE, Vol. 99, No. 1, pp.133-148.

Kaur, D.D. (2010) 'Smart grids and India', International Journal of Electrical Engineering \& Technology (IJEET), Vol. 1, No. 1, pp.157-164.

Liserre, M., Sauter, T. and Hung, J.Y. (2010) 'Future energy systems: integrating renewable energy sources into the smart power grid through industrial electronics', Industrial Electronics Magazine, Vol. 4, No. 1, pp.18-37, IEEE.

Misra, S., Krishna, P.V., Saritha, V., Agarwal, H., Shu, L. and Obaidat, M.S. (2013) 'Efficient medium access control for cyber physical systems with heterogeneous networks', IEEE System Journal, Vol. PP, No. 99, p.1.

Mohd, A. et al. (2008) 'Challenges in integrating distributed energy storage systems into future smart grid', IEEE International Symposium on Industrial Electronics, 2008, ISIE 2008, IEEE.

Molderink, A. et al. (2009) 'Domestic energy management methodology for optimizing efficiency in smart grids', PowerTech, IEEE, Bucharest.

Moslehi, K. and Kumar, R. (2010) 'A reliability perspective of the smart grid', IEEE Transactions on Smart Grid, Vol. 1, No. 1, pp.57-64.

Novosel, D. (2008) 'Emerging technologies in support of smart grids', Power and Energy Society General Meeting - Conversion and Delivery of Electrical Energy in the 21st Century, IEEE.

Sauter, T. and Lobashov, M. (2011) 'End-to-end communication architecture for smart grids', IEEE Transactions on Industrial Electronics, Vol. 58, No. 4, pp.1218-1228.

Tomich, J.L. (1998) Photonic Home Area Network Fiber/Power Insertion Apparatus, 7 July, U.S. Patent No. 5,778,116.

Tsoukalas, L.H. and Gao, R. (2008) 'From smart grids to an energy internet: assumptions, architectures and requirements', Third International Conference on Electric Utility Deregulation and Restructuring and Power Technologies, 2008, DRPT 2008, April IEEE, pp.94-98. 